\title{
The effect of position along the chain on the dynamic properties of hard chain segments
}

\author{
Julie A. McCormick, Carol K. Hall, ${ }^{\text {a) }}$ and Saad A. Khan \\ Department of Chemical Engineering, North Carolina State University, \\ Raleigh, North Carolina 27695-7905
}

(Received 13 December 2001; accepted 12 April 2002)

\begin{abstract}
Discontinuous molecular dynamics simulations are performed on systems containing 32 hard chains of length 192 at three volume fractions, $\phi=0.40,0.45$, and 0.50 , to investigate the effect of position on the segmental mean squared displacement. The mean squared displacements of various sized blocks of segments at different positions along the chain are calculated. First, the effect of block size on the dynamics of end and middle blocks is considered. It is found that small blocks provide a greater difference between the mean squared displacements of middle blocks, end blocks, and the whole chain than larger equal-sized blocks. Next, the portions of the chain exhibiting end and middle behavior are determined. It is found that a large portion of the chain displays middle behavior, while a small portion displays end behavior. Finally, the dynamics of segment relaxation along the chain are studied. The relaxation of small blocks of segments at different positions along the chain starts at the chain ends and progresses toward the chain middle with time, as the tube model predicts. The minor chain length, the portion of the chain that has relaxed, follows a power law with time, but the power is somewhat less than predicted. (c) 2002 American Institute of Physics. [DOI: 10.1063/1.1483295]
\end{abstract}

\section{INTRODUCTION}

One way to evaluate the ability of theories like the Rouse, ${ }^{1}$ reptation, ${ }^{2}$ and tube ${ }^{3}$ models to describe the dynamic behavior of entangled polymers is to test their predictions for the dependence of segmental dynamics on position along the chain against experimental data. Experiments ${ }^{4-6}$ designed to compare the dynamics of blocks of segments in the center of a chain with those at the chain ends have been performed but were limited to large blocks or relatively short times, raising the question of whether this is sufficient to obtain a complete picture of the effect of chain position on the diffusion of chain segments. Although some simulations have been performed to study segmental dynamics as a function of position along the chain, ${ }^{7-17}$ most $^{7-15}$ have focused on comparing the dynamics of segments in the chain middle to tube model predictions.

The aim of this study is to investigate the effect of the position along the chain on segmental dynamic behavior. We use discontinuous molecular dynamics computer simulations to study how the size of the block impacts conclusions drawn from comparing the dynamics of end segments to those of middle segments. The idea here is to see how small the blocks must be in order to distinguish the behavior of chain ends from that of the chain middle. Then, we determine the portion of the chain that exhibits chain middle behavior and the portions of the chain that exhibit chain end behavior. Finally, we investigate the dynamics of segment relaxation as a function of position along the chain to determine how the relaxation propagates along the chain.

Experimental studies ${ }^{4-6}$ comparing the dynamics of end

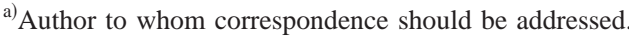

blocks to that of middle blocks have employed the techniques of neutron spin echo (NSE) spectroscopy, dynamic secondary ion mass spectroscopy, or specular neutron reflectivity. These techniques require the synthesis of labeled polymer samples in order to obtain dynamic information about specific sections along the chain. As described in detail below, the labeled end and middle blocks used in these studies have been relatively large, containing between $25 \%$ and $50 \%$ of the chain segments.

Ewen et al. ${ }^{4}$ performed NSE experiments to study segmental diffusion of entangled polydimethylsiloxane (PDMS) melts. Three separate samples of chains labeled by hydrogen-deuterium exchange were used to compare the motion of large middle chain sections $(37 \%)$, large end chain sections $(78 \%)$, and the entire chain. The tube diameter, $d_{T}$, which specifies the lateral constraints on the segments, was estimated for the chain middle, the chain ends, and the whole chain from the NSE experiments. They found that the lateral constraints were the same for the middle third of the chain, the outer third of the chain, and the whole chain.

Welp et al. ${ }^{5}$ investigated the interdiffusion of polystyrene (PS) chains during welding in the melt using dynamic secondary ion mass spectroscopy and specular neutron reflectivity to obtain information about the microscopic details of polymer dynamics. In this study, they used bilayer samples made up of two layers of oppositely labeled PS triblock chains to compare the diffusion behavior of the chain ends to that of the chain middle. In one layer, the inner $50 \%$ was deuterium labeled, and in the other layer, the outer $25 \%$ on each end was deuterium labeled. The samples were welded for various annealing times in order to obtain the deuterium peak height, peak position, and the area under the 
peak as a function of time. The Rouse model, ${ }^{1}$ the polymer mode-coupling model, ${ }^{18}$ and reptation theory ${ }^{2,3}$ provide unique predictions for these quantities. Reptation theory provided the best description of the interdiffusion results.

In a subsequent study, Welp et al. ${ }^{6}$ studied the motion of central and end sections of polymers independently during interdiffusion experiments by using different samples to observe the motion of each section directly. Specular neutron reflectivity was used to determine changes in the deuterium depth profile over time and thus the diffusion of a given chain section. Two different polymer bilayer samples were used to observe the motion of end sections and middle sections independently. Each bilayer sample contained a layer of hydrogenated-deuterated-hydrogentated PS (HDH-PS) that was 50\% hydrogenated PS and 50\% deuterated PS. The other layer in the ends bilayer sample contained fully deuterated PS (D-PS), and the other layer in the center bilayer sample contained fully hydrogenated PS (H-PS). The average penetration distances, $\langle X(t)\rangle$, of the ends and the centers were monitored. The experimental results were consistent with reptation predictions and inconsistent with Rouse predictions, the same as in previous work.

The many experimental challenges associated with determining the diffusion of selected segments along the length of polymer chains may explain the relatively limited body of experimental work in this area. First of all, there is a need for specialized equipment. Very few neutron spectrometers exist, and thus obtaining sufficient beam time to perform the experiments can be a challenge in itself. Secondly, tailored materials or multiple experimental techniques may be needed to access all the time and length scales of interest. Finally, obtaining information about the diffusion of specific segments along the chain requires the controlled synthesis of specially labeled polymers. This adds to the difficulty and cost of the experiments. An adequate portion of the material must be labeled to obtain a sufficient signal to noise ratio. Also, each chain section to be studied requires the synthesis of additional samples and the performance of additional experiments.

Computer simulation can provide new and complimentary information on the diffusion behavior of segments at different positions along the chain. Simulations do not require the preparation of a different system to study each section along the chain as is required during experiments; thus, a single simulation may provide information that would otherwise require many experiments. Also, following the motion of specific segments along the chain does not require that the chain be altered; in contrast, following the motion of specific polymer segments experimentally requires a chemical modification that may alter the dynamic behavior. Because of these advantages, computer simulation is a useful tool for studying the diffusion of segments at specific positions along the chain backbone.

Computer simulations yield detailed information about molecular motion. For this reason, a number of research groups have performed extensive simulations of entangled polymer melts ${ }^{7-17}$ to obtain information about the segmental diffusion behavior of chain molecules. The majority of these simulations have focused on comparing the dynamics of seg- ments in the chain middle to reptation and tube model predictions. ${ }^{7-15}$ A few simulations ${ }^{7,8,10}$ have compared the motion of the chain center to that of the chain ends, but most of the published results have been for relatively short times. Some simulation studies ${ }^{8-10,16,17}$ analyzed the dynamic behavior of segments at various positions along the length of the chain. Additional simulations are needed to characterize the segmental diffusion behavior of chain molecules completely.

A number of groups ${ }^{7-15}$ have performed computer simulations to see if the inner segment mean squared displacements of chain molecules agree with the tube model's $\mathrm{s}^{3,19}$ scaling predictions with time. A variety of different simulation methods have been employed, including lattice Monte Carlo, ${ }^{7-9}$ continuous molecular dynamics, ${ }^{10-12}$ and discontinuous molecular dynamics, ${ }^{13-15,17}$ all of which produced similar results. The inner segment mean squared displacements displayed a few different scaling regimes, but all of the tube model regimes were not attained.

A few researchers ${ }^{7,8,10}$ have compared the dynamic behavior of segments at the chain center to that of segments at the chain ends. Paul et al. ${ }^{7}$ compared the mean squared displacement of middle segments to that of end segments during their bond-fluctuation Monte Carlo simulations of chains of length $n=100$ at a cubic lattice fraction of $\phi=0.40$. During their molecular dynamics simulations, Kremer and Grest ${ }^{10}$ compared the mean squared displacement of the five outer segments to that of the five inner segments for chains of length $n=75$ and 150 . Both groups found that the mean squared displacement of the end monomers was greater than that of the center monomers.

Kreer et al. ${ }^{8}$ monitored the ratio of the outer monomer mean squared displacement to the inner monomer mean squared displacement for chain lengths between $n=16$ and 512 during their bond-fluctuation model Monte Carlo simulations. This ratio should start at 1 for early times, reach a maximum whose value depends on the dynamics model followed at intermediate times, and approach 1 at long times. For Rouse dynamics, the maximum value should be 2 , and for reptation, the maximum should approach $4 \sqrt{2}$ in the limit of infinite chain length. For all of the chain lengths that they studied, Kreer et al. found that the ratio started at approximately 1.5 at early times and approached 1 at long times. For short unentangled chains of length $n \leqslant 64$, the maximum was approximately 2, as expected for Rouse dynamics. For longer chains of length $n=128$, the maximum was approximately 3 , indicating increasing agreement with reptation dynamics. However, the chain lengths studied were not long enough to reach the true reptation predictions.

Some groups ${ }^{8-10,16,17}$ have studied the dynamic behavior of segments at various positions along the backbone of chain molecules. Kreer et $\mathrm{l}^{8}{ }^{8}$ studied the relaxation of the bond vector at different positions along the chain. They plotted the bond vector autocorrelation function, $\phi_{n}(t)$, versus position along the chain for their simulation results and for Rouse model predictions at two different chain lengths, $n=16$ and 128 . The bond vector autocorrelation function, $\phi_{n}(t)$, represents the probability that the original orientation of the bond vector at segment position $n$ is still present at time, $t$. For 
both chain lengths, the bond vectors near the chain ends relaxed faster than those in the middle. However, the inner bond vectors of the longer chains relaxed more slowly than those of the shorter chains. Both systems exhibited deviations from Rouse behavior, but the deviations were greater for the longer chains than for the shorter chains.

Kolinski and co-workers ${ }^{16,9}$ analyzed the mean squared displacement of individual segments at all positions along the chain contour for chains of length $n=216$ on a diamond lattice. They compared their simulation results to Rouse-type equations that fit the data fairly well, except for noticeable deviations at the chain ends which increased with increasing time. The Rouse-type equations underestimated the increased mobility of the chain ends compared to the chain middle. They concluded that their chain motion was similar to that of a modified Rouse chain with an increased self-diffusion coefficient due to a significantly decreased friction constant for the end segments.

Kremer and $\mathrm{Grest}^{10}$ determined the mean squared displacement of chain blocks containing five segments each at different positions along chains of length $n=150$ during their molecular dynamics simulations. Blocks of segments relaxed at different times depending on their position along the chain. At a block's relaxation time, its mean squared displacement became greater than that of the middle block. The enhanced mobility of the end block began at early times. As time increased, the blocks closer toward the chain middle began to relax.

In a previous study of entanglement relaxation and release, ${ }^{17}$ we reported the mean squared displacement of blocks of segments at different positions along chains of length $n=192$ at three volume fractions, $\phi=0.40,0.45$, and 0.50 . The mean squared displacements of various blocks of 10 segments along the chain were analyzed to determine both the progression of relaxation along the chain and the actual longest relaxation time without employing assumptions based on specific theories. During early times, the chain molecules' relaxation started at the chain ends and progressed toward the chain middle as conjectured by the tube model. However, this trend did not persist throughout the relaxation, but instead reversed so that final relaxation occurred at the end segments as expected for the release of knots instead of at the middle segments as predicted by the tube model. The actual final relaxation time, as determined from the mean squared displacement behavior, occurred at times that were longer than $\tau_{d}$ predictions obtained using tube model methods. This suggested that another method of entanglement release, possibly that of interchain entanglements, was present in the simulation systems.

In this paper, we extend our previous work ${ }^{17}$ to include a more thorough analysis of the effect of segment position along the chain on the dynamic properties. We have performed discontinuous molecular dynamics simulations on three systems: 32 hard chain molecules of chain length 192 at three volume fractions, $\phi=0.40,0.45$, and 0.50 . The mean squared displacements of various blocks of segments along the chain were calculated over the course of the simulations. First, we determine how the size of the blocks considered impacts conclusions drawn from comparing the dy- namics of end segments and middle segments. This is done by calculating the mean squared displacements averaged over blocks of segments in the chain middle, at the chain ends, and over the whole chain for two cases, the triblock case and the small block case. In the triblock case, the chain is sectioned into three equal-sized blocks of segments, so that the middle block contains 64 segments in the chain middle, and the end blocks contain 64 segments on each chain end. In the small block case, the middle block contains 10 segments in the chain middle, and the end blocks contain 5 segments on each chain end. Then, we calculate the mean squared displacements of middle and end blocks of various sizes to determine what portion of the chain exhibits chain middle behavior and what portions of the chain exhibit chain end behavior. Finally, we calculate the mean squared displacements for small blocks of segments at various positions along the chain to ascertain how the relaxation propagates along the chain. The relaxation dynamics are determined by estimating the minor chain length and its power-law scaling with time.

Highlights from our simulation results are the following. The small-block case provides a greater contrast between the mean squared displacements of the middle block, the end blocks, and the whole chain than the triblock case provides. Comparing the triblock and small-block cases directly reveals that the mean squared displacement for the end blocks in the small-block case is larger than in the triblock case, but the mean squared displacements for the middle blocks are the same in both cases. Analysis of the mean squared displacements of different sized end and middle blocks reveals that a large portion of the chain exhibits chain middle behavior, and a small portion of the chain exhibits chain end behavior. The relaxation of small blocks of segments at different positions along the chains starts at the chain ends and propagates toward the chain middle, as the tube model predicts. The minor chain length, the portion of the chain that has relaxed, follows a power law with time, but increases somewhat more slowly than predicted.

The remainder of the paper is organized as follows: Section II describes the molecular model and simulation techniques and presents the static properties of the chains. Section III provides relevant background information on chain segment dynamics. Section IV describes our simulation results. A brief summary of our conclusions and some further discussion are given in Sec. V.

\section{MOLECULAR MODEL AND SIMULATION METHODS}

In this study, a polymer fluid is modeled as a system of hard chains and simulated via discontinuous molecular dynamics (DMD), the technique used by Smith et al. ${ }^{20}$ In this section, we describe the molecular model for the chain molecules, review the DMD technique applied to this model, and present the static properties of the systems.

Because polymers are large molecules, their macroscopic behavior is dictated more by molecular size and geometry than by chemical structure. This suggests that the main features of polymers that must be incorporated into a model are the chain connectivity, flexibility, and the excluded volume of the segments. To model these features, Flory ${ }^{21}$ 
proposed the tangent hard sphere chain model. In order to facilitate molecular dynamics simulation, Rapaport ${ }^{22}$ allowed the bond length, $l$, to vary over the range $\sigma<l<(1+\delta) \sigma$, where $\delta$ is a small fraction. In effect, the segments of the chain are joined by short strings of length $\delta \sigma$. Bellemans et $a .^{23}$ modified the Rapaport model to allow adjacent segments of a chain to interpenetrate by the length of the strings so that $(1-\delta) \sigma<l<(1+\delta) \sigma$.

All simulations in this study employed the hard chain model with the Bellemans et al. bonding constraints. The potential of interaction $U(r)$ between two nonbonded segments in the same or different chains is given by the hard sphere potential,

$$
U(r)=\left\{\begin{array}{cc}
\infty, & r \leqslant \sigma \\
0, & r>\sigma
\end{array}\right.
$$

where $r$ is the distance between two segments, and $\sigma$ is the segment diameter. The potential energy between two bonded segments in a chain is given by the Bellemans et al. potential,

$$
U(r)= \begin{cases}\infty, & r \leqslant(1-\delta) \sigma \\ 0, & (1-\delta) \sigma<r<(1+\delta) \sigma \\ \infty, & r \geqslant(1+\delta) \sigma\end{cases}
$$

In this work, the bond extension parameter, $\delta$, was set to 0.1 , since this provides sufficient displacement of the molecules while prohibiting chain crossing.

The simulations were performed using the discontinuous molecular dynamics technique used by Smith et al. ${ }^{20}$ for hard chains. This method is essentially an extension of the DMD method developed by Alder and Wainwright ${ }^{24-25}$ (and discussed by Haile $^{26}$ ) for hard spheres to include bond stretch events. An event, or collision, occurs when the distance between two segments becomes equal to a discontinuity in the potential. Thus, the chains experience two possible types of events, core collisions (any two segments coming into contact) and bond stretches (two bonded segments reaching their maximum separation distance). The DMD technique for simulating systems with discontinuous potentials takes advantage of the fact that linear trajectories between collisions produce equations of motion that can be solved analytically at successive collisions.

The simulations develop on a collision-by-collision basis by locating the next event in the system (core collision or bond extension), advancing the system to that point in time, computing the collision dynamics, and repeating the process. Possible events, core collisions and bond stretches, are investigated to determine the time when they will occur. A schedule of collision times is maintained to determine the sequence of events over the course of the simulation. Once the next event is determined, all the segments are advanced until that event occurs. The elastic event between two segments changes their velocities. Since the velocities of the colliding particles change, their paths and thus their future collision partners will also change. Updating the collision lists requires the calculation of new collision times and partners for
TABLE I. Size and length of the simulated systems. ${ }^{a}$

\begin{tabular}{ccccc}
\hline \hline$n$ & $N_{c}$ & $\phi$ & Collisions $\left(10^{9}\right)$ & $t^{*}\left(10^{6}\right)$ \\
\hline 192 & 32 & 0.40 & 20 & 0.311 \\
& 32 & 0.45 & 82 & 1.03 \\
& 32 & 0.50 & 200 & 1.92 \\
\hline \hline
\end{tabular}

${ }^{\text {a }}$ The sizes of the simulated systems are given in terms of the chain length, $n$, and the number of chains, $N_{c}$. Other simulation parameters are the packing fraction, $\phi$, the number of collisions reported in billions of events, and the total reduced simulation time, $t^{*}$.

the colliding segments after each event; this is the most computationally intensive part of a discontinuous molecular dynamics simulation.

Because of the great computational demand associated with calculating new collision times, considerable effort has focused on improving the efficiency of this part of the simulation. The time needed to update the collision and partner lists is decreased by using a number of efficiency techniques as discussed in detail by Smith et al. ${ }^{20}$ including neighbor lists, ${ }^{27}$ linked lists, ${ }^{27}$ binary trees, ${ }^{28}$ and false positioning. ${ }^{29}$ The use of these techniques has allowed us to simulate systems of long chain molecules over more than six orders of magnitude in reduced time.

Discontinuous molecular dynamics simulations were performed on three systems: 32 chains of length 192 at three different volume fractions, $\phi=0.40,0.45$, and 0.50 . The volume fraction is the fraction of the system volume occupied by chain segments and is defined as $\phi \equiv \pi N \sigma^{3} / 6 V$, where $N$ is the total number of chain segments, and $V$ is the volume of the simulation cell. The simulations were performed in a cubic simulation cell with standard periodic boundary conditions.

Starting configurations at the different volume fractions studied were obtained by generating a single initial configuration at a low volume fraction $(\phi=0.28)$ and then increasing the volume fraction to the desired value. The initial configuration was generated by constructing each chain from a random walk with a bond length of $(1+\delta / 2) \sigma$ and assigning random velocities to each segment using a Gaussian number generator. After an initial configuration was obtained, the velocities were scaled to obtain an average segment velocity of $\mathbf{v}=\mathbf{0}$ and a reduced system temperature of $k_{B} T=1.0$. The volume fraction of each system was then increased by growing segment diameters at bond stretch events ${ }^{20}$ while performing the discontinuous molecular dynamics simulation with the nonoverlapping Rapaport model mentioned above. The starting configurations at $\phi=0.40,0.45$, and 0.50 were obtained when the volume fractions of interest were reached. Each of the three starting configurations was then relaxed using the Bellemans et al. model during the equilibration phase. The equilibration phase was finished, and the production phase was started once the chain center of mass moved at least one radius of gyration (on average).

Table I summarizes the systems studied and includes the chain length, $n$, the number of chains, $N_{c}$, the volume fraction, $\phi$, the number of collisions, and the total times sampled during the production phases of the simulations. The simulation times are presented throughout the paper in terms of 
TABLE II. Static properties of the systems. ${ }^{\mathrm{a}}$

\begin{tabular}{cccccc}
\hline \hline$n$ & $\phi$ & $\left\langle R^{2}\right\rangle$ & $\left\langle R_{g}^{2}\right\rangle$ & $\left\langle R^{2}\right\rangle /\left\langle R_{g}^{2}\right\rangle$ & $Z$ \\
\hline 192 & 0.40 & $379.68( \pm 28.13)$ & $61.72( \pm 2.70)$ & 6.15 & $761.71( \pm 0.14)$ \\
& 0.45 & $331.69( \pm 36.08)$ & $55.36( \pm 3.98)$ & 5.99 & $1127.80( \pm 0.18)$ \\
& 0.50 & $317.44( \pm 35.23)$ & $52.07( \pm 4.49)$ & 6.10 & $1665.06( \pm 0.23)$ \\
\hline \hline
\end{tabular}

${ }^{a}$ The mean squared end-to-end distance, $\left\langle R^{2}\right\rangle$, the mean squared radius of gyration, $\left\langle R_{g}^{2}\right\rangle$, the ratio of the sizes, $\left\langle R^{2}\right\rangle /\left\langle R_{g}^{2}\right\rangle$, and the compressibility factor, $Z$, are reported. The chain sizes are reported in units of $\sigma^{2}$. Values in parentheses are the standard deviations.

reduced units, $t^{*}=\left(k_{B} T / m \sigma^{2}\right)^{1 / 2} t$, where $k_{B}$ is the Boltzmann constant, $T$ is the temperature, and $m$ is the mass of a segment. All simulations were performed at a reduced temperature of $k_{B} T=1.0$ and a mass of $m=1.0$ for all segments.

The static properties of the systems studied, including the mean squared end-to-end distance, $\left\langle R^{2}\right\rangle$, the mean squared radius of gyration, $\left\langle R_{g}^{2}\right\rangle$, and the compressibility factor, $Z$, were monitored over the course of the simulations to confirm fluid equilibration. The mean squared end-to-end distance was calculated from $\left\langle R^{2}\right\rangle \equiv\left\langle\left(\mathbf{r}_{1}-\mathbf{r}_{n}\right)^{2}\right\rangle$, where $\mathbf{r}_{1}$ and $\mathbf{r}_{n}$ are the coordinates of the first and last chain segments and \langle\rangle denotes an ensemble average. The radius of gyration was calculated from $\left\langle R_{g}^{2}\right\rangle \equiv\left\langle\sum_{i=1}^{n}\left(\mathbf{r}_{i}-\mathbf{r}_{\mathrm{c} . \mathrm{m} .}\right)^{2}\right\rangle / n$, where $\mathbf{r}_{i}$ is the position of segment $i$ and $\mathbf{r}_{\mathrm{c} . \mathrm{m} .}=(1 / n) \sum_{i=1}^{n} \mathbf{r}_{i}$ is the position of the chain center of mass. For a Gaussian chain, both the end-to-end distance and the radius of gyration should scale linearly with chain length so that the ratio $\left\langle R^{2}\right\rangle /\left\langle R_{g}^{2}\right\rangle$ $\approx 6$. $^{21}$ The compressibility factor was calculated from the Clausius virial theorem: $Z=n-\left(m \Sigma_{\text {coll }} \mathbf{r}_{i j} \cdot \Delta \mathbf{v}_{i j}\right) /$ $\left(3 N_{c} k_{B} T t_{e}\right)$, where $\mathbf{r}_{i j}$ is the vector between segment centers at a collision, $\Delta \mathbf{v}_{i j}$ is the velocity change for the colliding pair, $N_{c}$ is the number of chains in the system, and $t_{e}$ is the elapsed simulation time over which the sum is calculated.

The results for the static properties, $\left\langle R^{2}\right\rangle,\left\langle R_{g}^{2}\right\rangle$, $\left\langle R^{2}\right\rangle /\left\langle R_{g}^{2}\right\rangle$, and $Z$ are presented in Table II. The standard deviations in $\left\langle R^{2}\right\rangle,\left\langle R_{g}^{2}\right\rangle$, and $Z$ were determined from the average values for batches of the simulations containing 2 billion collisions each. The values obtained for $\left\langle R^{2}\right\rangle /\left\langle R_{g}^{2}\right\rangle$ indicate that the chains used in this study are essentially Gaussian. The compressibility factor was found to be linear with chain length and consistent with the work of Zhou et al. ${ }^{30}$ Agreement between the static properties of the simulations and predicted behavior confirms the equilibration of the systems. With the confirmation of fluid equilibration, the production phases of the simulations were started in order to analyze the dynamic properties of the systems.

During the course of the simulations, the coordinates of all chain segments were stored so that they could be used to determine the dynamic properties. These coordinates were later analyzed to obtain the mean squared displacement results presented in this paper.

\section{CHAIN DYNAMIC PROPERTY CALCULATIONS}

Microscopic information about polymer diffusion is obtained by analyzing the time dependence of chain and chain segment displacements. The atomic mean squared displacement, $g(t)$, is the average squared distance a chain segment moves after a time, $t$, and is defined as

$$
g(t) \equiv \frac{1}{n} \sum_{i=1}^{n}\left\langle\left|\mathbf{r}_{i}\left(t+t_{0}\right)-\mathbf{r}_{i}\left(t_{0}\right)\right|^{2}\right\rangle
$$

where $\mathbf{r}_{i}(t)$ is the position of atom $i$ at time $t$, the sum is over all chain segments $i$, and \langle\rangle represents the ensemble average that is over all time origins $t_{0}$ as well as over all molecules. Equation (3) provides the average mean squared displacement of all segments within the chains.

The average mean squared displacement for specific blocks of the chain can be obtained by restricting this calculation to particular segments of the chain. For example, we have calculated the mean squared displacement for middle blocks of the chain and for end blocks of the chain. This was done by averaging over only those segments of interest. The middle block mean squared displacement averaged over $k$ middle segments is calculated from

$$
g_{\text {middle }}(t) \equiv \frac{1}{k} \sum_{i=n / 2+1-k / 2}^{n / 2+k / 2}\left\langle\left|\mathbf{r}_{i}\left(t+t_{0}\right)-\mathbf{r}_{i}\left(t_{0}\right)\right|^{2}\right\rangle
$$

Likewise, the end block mean squared displacement averaged over $k$ segments on each chain end is calculated from

$$
\begin{aligned}
g_{\text {end }}(t) \equiv & \frac{1}{2 k}\left(\sum_{i=1}^{k}\left\langle\left|\mathbf{r}_{i}\left(t+t_{0}\right)-\mathbf{r}_{i}\left(t_{0}\right)\right|^{2}\right\rangle\right. \\
& \left.+\sum_{i=n-(k-1)}^{n}\left\langle\left|\mathbf{r}_{i}\left(t+t_{0}\right)-\mathbf{r}_{i}\left(t_{0}\right)\right|^{2}\right\rangle\right) .
\end{aligned}
$$

Throughout the paper, the mean squared displacements are reported in reduced units by dividing by the diameter squared, $\sigma^{2}$.

The tube model ${ }^{19,3}$ predicts that an entangled chain exhibits four distinct types of motion as characterized by the scaling of the atomic mean squared displacement, $g(t)$, with time. These four different types of motion are (1) $g \sim t^{1 / 2}$ for $t<\tau_{e}$, where $\tau_{e}$ is the entanglement time, (2) $g \sim t^{1 / 4}$ for $\tau_{e}$ $<t<\tau_{R}$, where $\tau_{R}$ is the Rouse time, (3) $g \sim t^{1 / 2}$ for $\tau_{R}<t$ $<\tau_{d}$, where $\tau_{d}$ is the disengagement time, and (4) $g \sim t$ for $t>\tau_{d}$. In our previous work, ${ }^{17}$ the entanglement times were estimated to be $\tau_{e} \approx 1800,3000$, and 4500 for the chains of length 192 at the volume fractions of $\phi=0.40,0.45$, and 0.50 . The Rouse times were estimated to be $\tau_{R} \approx 25000$, 67000 , and 100000 at the volume fractions of $\phi=0.40$, 0.45 , and 0.50 . The disengagement times could not be determined conclusively. 


\section{RESULTS AND DISCUSSION}

In this section, we present and discuss the results from our molecular dynamics simulations. First, we investigate how the size of the block considered affects conclusions about the difference between the dynamics of end segments and middle segments of chain molecules. Next, we determine which portion of the chain exhibits chain middle behavior and which portions exhibit chain end behavior. Finally, we investigate the dynamics of segment relaxation at different positions along the chain to ascertain how the relaxation propagates along the chain length.

\section{A. Effect of block size on the difference between the dynamics of end and middle blocks}

It is of interest to determine if large blocks are able to distinguish the behavior of chain ends from that of the chain middle. Experimental studies have been limited to considering the dynamics of relatively large end and middle blocks containing between $25 \%$ and $50 \%$ of the chain segments. ${ }^{4-6}$ In contrast, simulation studies have investigated the dynamics of individual segments or small blocks containing up to 10 segments. ${ }^{7-17}$ In this study, we use both approaches to compare the mean squared displacement behavior of end and middle chain segments.

\section{Triblock analysis}

In this part of our study, each chain is divided into thirds and then the mean squared displacements are calculated by averaging over the chain end blocks, the chain middle block, and the whole chain. This analysis is similar to experiments performed on labeled triblock polymers made up of equal sized blocks.

Figure 1 displays the mean squared displacements of the chain end sections, the chain middle section, and the whole chain for chains of length 192 at three different volume fractions, (a) $\phi=0.40$, (b) $\phi=0.45$, and (c) $\phi=0.50$. The top curve in each graph corresponds to the mean squared displacement of the end sections (segments 1-64 and 129192). The middle curve in each graph corresponds to the mean squared displacement averaged over all chain segments. The bottom curve in each graph corresponds to the mean squared displacement of the middle section (segments 65-128).

For the triblock case, the mean squared displacements of the end blocks, the whole chain, and the middle blocks have similar values for each system. This is especially true at early times, $t \leqslant \tau_{e}$, when the three different mean squared displacements are essentially identical. As time increases past $\tau_{e}$, a detectable difference is observed between the three different mean squared displacements. The whole chain mean squared displacements are lower than the end block mean squared displacements but higher than the middle block mean squared displacements. This occurs because the end segments become more relaxed than the middle segments and thus experience more mobility than the rest of the chain. At the same time, the middle segments remain confined and thus experience less mobility than the rest of the chain. As time increases further toward the longest times
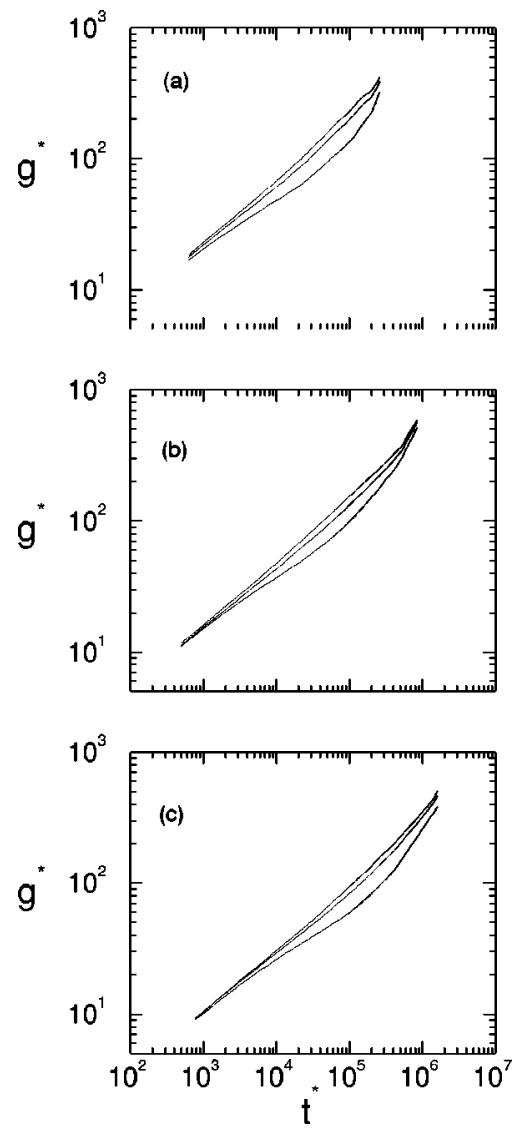

FIG. 1. Reduced mean squared displacement vs reduced time for the chains of length 192 at (a) $\phi=0.40$, (b) $\phi=0.45$, and (c) $\phi=0.50$. Included, from top to bottom, are the mean squared displacements of the end blocks, the whole chain, and the middle block for the triblock case.

reported, the three different mean squared displacements have similar values again as segments at all positions along the chain have relaxed.

The early-time simulation results are in qualitative agreement with the results from the NSE experiments of Ewen et al. ${ }^{4}$ In their study of labeled triblock PDMS chains containing equal sized blocks, Ewen et al. found that the normalized coherent intermediate scattering function was essentially the same for the end and middle segments as it was for the whole chain. The NSE experiments were only able to access relatively short times, less than the Rouse time, which may not be long enough to reach the times when the greatest difference between the motion of the end and middle sections occurs. Therefore, longer experimental times or smaller labeled sections may be needed to observe a distinct difference between the behavior of the end and middle sections.

\section{Small-block analysis}

The small-block analysis was performed to further probe the difference between the mean squared displacements of the outermost segments and the innermost segments. In this part of the study, we present the mean squared displacements for end blocks that contain five segments on each chain end and middle blocks that contain ten segments in the chain 

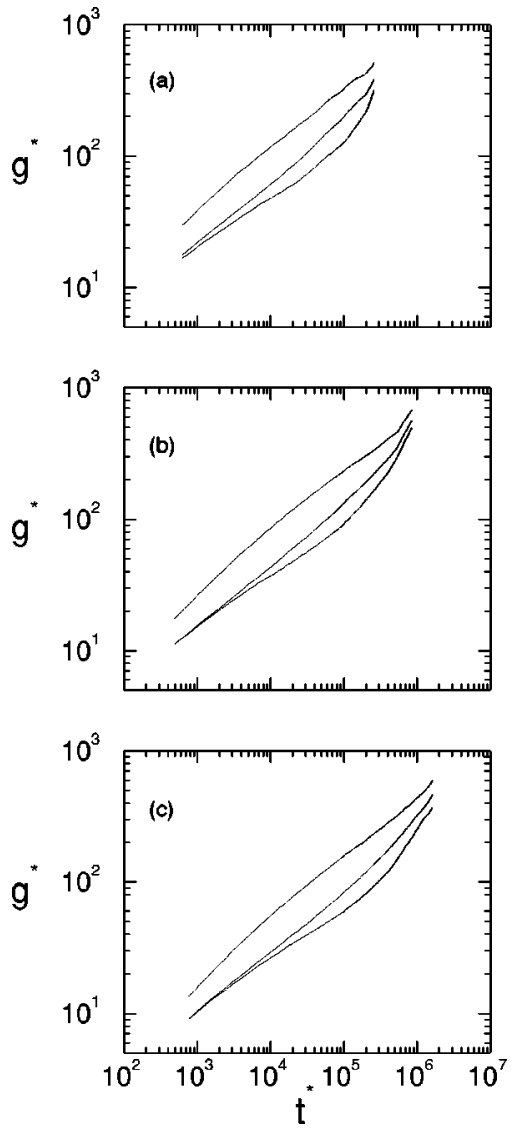

FIG. 2. Reduced mean squared displacement vs reduced time for the chains of length 192 at (a) $\phi=0.40$, (b) $\phi=0.45$, and (c) $\phi=0.50$. Included, from top to bottom, are the mean squared displacements of the end blocks, the whole chain, and the middle block for the small-block case.

middle. These blocks of segments are small enough to concentrate the analysis to specific parts of the chain but large enough to obtain good statistics.

Figure 2 displays the mean squared displacement averaged over the chain end sections, the chain middle section, and the whole chain for chains of length 192 at (a) $\phi$ $=0.40$, (b) $\phi=0.45$, and (c) $\phi=0.50$. As in the triblock case (and as expected), the top curve is for the mean squared displacements averaged over the outer segments, the middle curve is for that averaged over all segments, and the bottom curve is for that averaged over the middle segments. In the small block case, the end sections include segments $1-5$ and 188-192, and the middle sections include segments 92-101.

For the small-block case, the mean squared displacements of the end blocks are significantly different from those of the whole chain and those of the middle blocks for all of the systems studied. At the earliest times shown, $t \leqslant \tau_{e}$, the mean squared displacements averaged over the middle sections are essentially the same as those averaged over the whole chain, while those averaged over the end sections are much larger. This indicates that the endmost segments are more mobile than the rest of the chain. As time increases past $\tau_{e}$, the mean squared displacement curves of the middle blocks separate from those of the whole chain, indicating decreased mobility and increased confinement of the middle sections. At longer times, all of the mean squared displace-
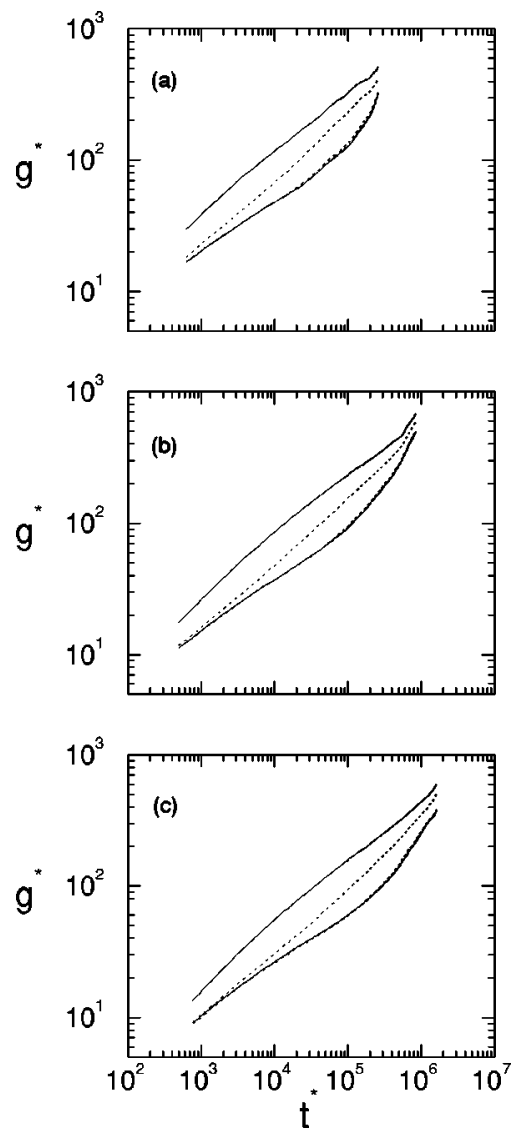

FIG. 3. Reduced mean squared displacement vs reduced time for the chains of length 192 at (a) $\phi=0.40$, (b) $\phi=0.45$, and (c) $\phi=0.50$. The mean squared displacements of the end blocks and middle blocks for the smallblock case are represented by solid lines. The mean squared displacements of the end blocks and middle blocks for the triblock case are represented by dotted lines.

ment curves approach each other as all of the segments of the chains relax.

There is a larger separation between the different mean squared displacement curves in the small-block case than there was in the triblock case. The difference between the mean squared displacements of the end and middle blocks is much greater here than it was for the triblock case.

\section{Comparison between the triblock analysis and the small-block analysis}

The differences between the results from the equal sized triblock case and those from the small-block case can be seen more clearly by comparing the mean squared displacement results obtained for the end and middle chain sections from the two methods on the same graph.

Figure 3 displays the mean squared displacement results for the end and middle blocks from both the triblock and small-block cases for chains of length 192 at (a) $\phi=0.40$, (b) $\phi=0.45$, and (c) $\phi=0.50$. The results for the triblock case are represented by dotted lines, and the results for the small-block case are represented by solid lines. In each graph, the top curve is the end section mean squared displacement for the small-block case. The second curve from the top is always the end section mean squared displacement 
for the triblock case. The bottom two curves are always the middle section mean squared displacements for the triblock and small-block cases.

There is a large difference between the end section mean squared displacements from the triblock and small-block cases over all of the times studied, but the difference is smaller at long times. The middle section mean squared displacements are essentially the same in the triblock case as in the small-block case over all times. This indicates that a larger portion of the chain has middle section behavior than has end section behavior. Therefore, small blocks must be used to probe the end section behavior, but larger blocks can be used to probe the middle section behavior.

The ratio of the end block to middle block mean squared displacement was calculated for the different cases. This ratio can be used to determine the difference in the mobility of the chain ends and the chain middle and to compare the ability of the triblock and small-block studies to capture this difference. The ratio was also calculated for the end segment on each chain end and the two middle segments to compare the results for the triblock and small-block cases to the limiting case, which is expected to reveal the actual behavior of the middle and end segments. The maximum value can also be compared to the Rouse model and reptation predictions, as was done by Kreer et al. ${ }^{8}$ to determine which model is better able to capture the dynamics of the different chain sections.

Figure 4 displays the ratio of the end block mean squared displacement to the middle block mean squared displacement for both the triblock and small-block cases for chains of length 192 at (a) $\phi=0.40$, (b) $\phi=0.45$, and (c) $\phi=0.50$. Also included for comparison is the ratio of the end segment (segments 1 and 192) to middle segment (segments 96 and 97) mean squared displacement. The top (dot-dashed) curve in each graph is for the limiting case, the middle (solid) curve is for the small-block case, and the bottom (dotted) curve is for the triblock case.

The ratio of the end block mean squared displacement to the middle-block mean squared displacement provides a good measure of the difference between the dynamics of the different size blocks considered. The small-block case captures the difference in mobility between the chain end segments and the chain middle segments much better than the triblock case. The ratio for the small-block case is very close to the limiting case; whereas, the ratio for the triblock case is much smaller and does not change as much over time. Small blocks, or the limiting segments, are needed to compare the maximum in this ratio to the theoretically predicted value, because the maximum in the triblock case is dramatically smaller than in the limiting case. For chains of length 192, the maximum in the ratio for the triblock case is approximately $1.6-1.7$, whereas for the other cases it is approximately $2.6-2.8$. The maximum value for the triblock case is less than the Rouse prediction of 2; the maximum values for the other cases are greater than the Rouse prediction but less than the reptation prediction of $4 \sqrt{2}$ (5.656). The large blocks in the triblock case are too large to capture the true dependence of segment position along the chain on the dynamic behavior, and thus smaller sections should be used.
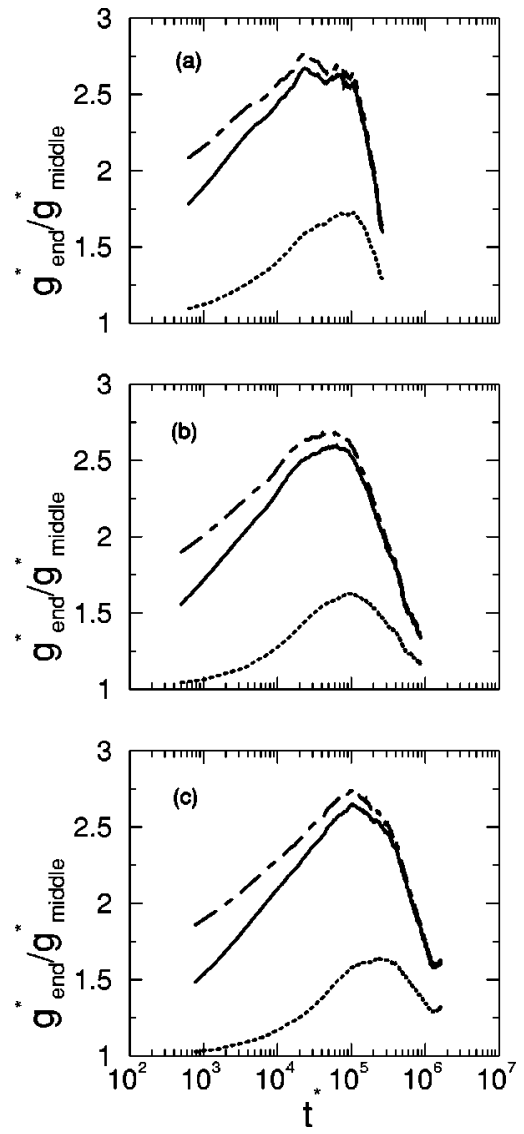

FIG. 4. Ratio of the end section mean squared displacement to the middle section mean squared displacement vs reduced time for chains of length 192 at (a) $\phi=0.40$, (b) $\phi=0.45$, and (c) $\phi=0.50$. The ratios for the limiting case are represented by dot-dashed lines, the ratios for the small-block case are represented by solid lines, and the ratios for the triblock case are represented by dotted lines.

\section{B. Portion of segments exhibiting chain end and chain middle behavior}

In this section we determine what portion of the chain exhibits middle behavior and what portion exhibits end behavior. The aim is to determine what size blocks are sufficient to capture the middle section and end section behavior, as measured by the mean squared displacements.

\section{Portion of segments exhibiting chain middle behavior}

The mean squared displacements of different sized blocks of middle segments were analyzed to determine how large the middle blocks can be and still display primarily middle section behavior.

Figure 5 displays the mean squared displacements of blocks of middle segments of different sizes as a function of reduced time for chains of length 192 at (a) $\phi=0.40$, (b) $\phi=0.45$, and (c) $\phi=0.50$. The middle block sizes are 2,10 , $64,96,128$, and 172 segments. Most of these mean squared displacement curves are the same as that for the smallest middle block containing two segments (bottom curve). The ability of the different sized middle blocks to mimic the behavior of the middle segments depends on the time range of interest. At the early times, $t \leqslant \tau_{e}$, there is no detectable difference between the mean squared displacements of the 

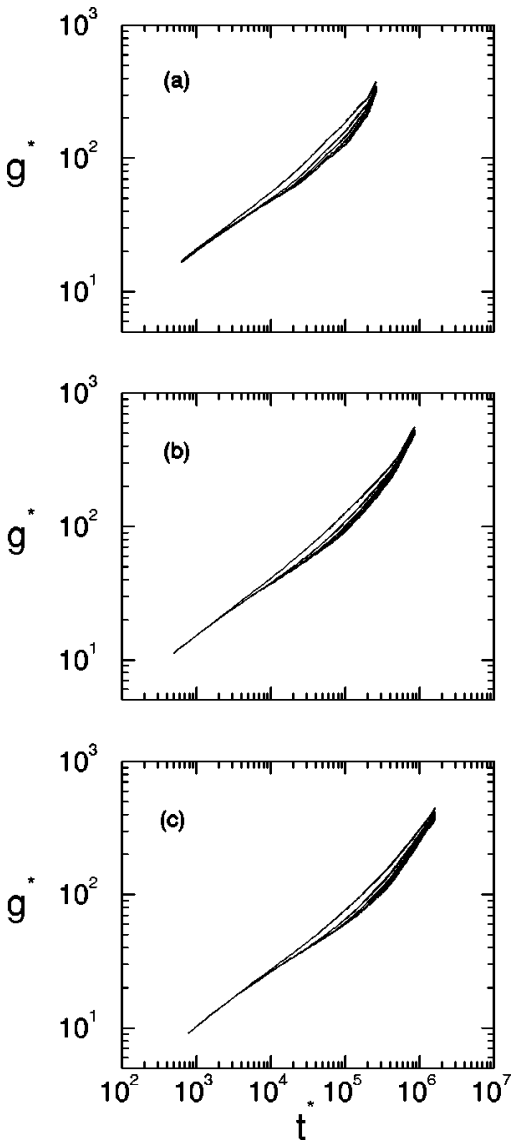

FIG. 5. Reduced mean squared displacement of middle blocks vs reduced time for chains of length 192 at (a) $\phi=0.40$, (b) $\phi=0.45$, and (c) $\phi$ $=0.50$. Included are the mean squared displacements for middle blocks containing $172,128,96,64,10$, and 2 segments, from top to bottom.

different sized middle blocks. During intermediate times, $t$ $\approx \tau_{R}$, the mean squared displacements of the three largest middle blocks studied, containing 96, 128, and 172 segments, are larger than those of the smaller middle blocks. This suggests that the segments in these larger blocks that are closer to the ends have started relaxing by this time. At longer times, the mean squared displacements of the different sized blocks approach the same curve again.

From the mean squared displacement curves of the different sized middle blocks, it appears that the middle blocks containing 2, 10, and 64 segments provide similar behavior. The mean squared displacements of the larger middle blocks (96, 128, and 172 segments) exhibit some deviation from that of the middle segments during the intermediate times around $\tau_{R}$. To determine the extent of the deviation between the different sized middle blocks, the fractional difference between the mean squared displacement of each sized block and that of the smallest block containing two segments was calculated and analyzed.

Figure 6 displays the fractional difference between the mean squared displacements of the different sized middle blocks $(10,64,96,128$, and 172 segments) and that of the reference middle block containing two segments as a function of reduced time for chains of length 192 at (a) $\phi$ $=0.40$, (b) $\phi=0.45$, and (c) $\phi=0.50$. The curves from top to bottom are for blocks containing $172,128,96,64$, and 10
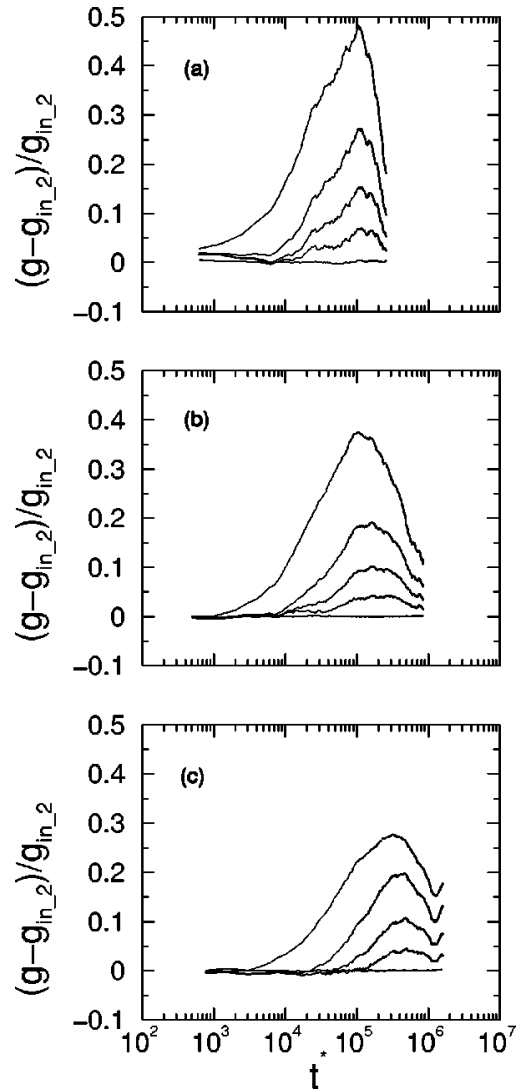

FIG. 6. Fractional difference between the mean squared displacement of different sized middle blocks and that of the reference middle block containing two segments vs reduced time for chains of length 192 at (a) $\phi$ $=0.40$, (b) $\phi=0.45$, and (c) $\phi=0.50$. Included are the fractional differences for middle blocks containing 10, 64, 96, 128, and 172 segments, from bottom to top.

segments. Over the whole time range, the fractional difference for the block containing 10 segments is approximately zero. At early times, the fractional differences for the other sized blocks is small, and some have smaller mean squared displacements than the two-segment reference block. The reference block should have the smallest mean squared displacement at all times. Although this is not observed, the most negative fractional difference, -0.01 , is probably not significantly different than 0 . As time increases, the fractional differences for the larger blocks increase. The maximum value in the fractional difference provides a measure of the different sized middle blocks' ability to mimic the behavior of the middle segments.

Table III lists the maximum fractional difference between the mean squared displacement of the different sized middle blocks and that of the reference middle block containing two segments. The values are largest for the chains at the smallest volume fraction, $\phi=0.40$, and similar for the chains at the other two volume fractions, $\phi=0.45$ and 0.50 . This indicates that the chains are more confined, and more segments exhibit middle behavior at the larger volume fractions. For any given volume fraction, the maximum fractional difference increases with increasing middle block size as expected. The maximum fractional difference is less than 0.11 for the middle blocks containing 10 segments and 64 segments at all volume fractions and for the middle blocks 
TABLE III. Maximum fractional difference for the different sized middle blocks. ${ }^{\text {a }}$

\begin{tabular}{cccccc}
\hline \hline & \multicolumn{5}{c}{ Number of segments } \\
\cline { 2 - 6 }$\phi$ & 10 & 64 & 96 & 128 & 172 \\
\hline 0.40 & 0.0051 & 0.0706 & 0.1545 & 0.2734 & 0.4823 \\
0.45 & 0.0026 & 0.0437 & 0.1030 & 0.1924 & 0.3754 \\
0.50 & 0.0038 & 0.0448 & 0.1076 & 0.1987 & 0.2774 \\
\hline \hline
\end{tabular}

${ }^{a}$ The maximum fractional differences between the mean squared displacement of different sized middle blocks and that of the two middle segments are reported. The different sized middle blocks include 10, 64, 96, 128, and 172 segments in the chain middle.

containing 96 segments at the higher volume fractions, $\phi$ $=0.45$ and 0.50 . This indicates that more of the chain is confined, and thus more segments exhibit middle behavior at the higher volume fractions.

\section{Portion of segments exhibiting chain end behavior}

The mean squared displacements of different sized blocks of end segments were analyzed to determine how large the end blocks can be and still display primarily end section behavior. Figure 7 displays the mean squared displacements of blocks of end segments of different sizes as a function of reduced time for chains of length 192 at (a) $\phi$
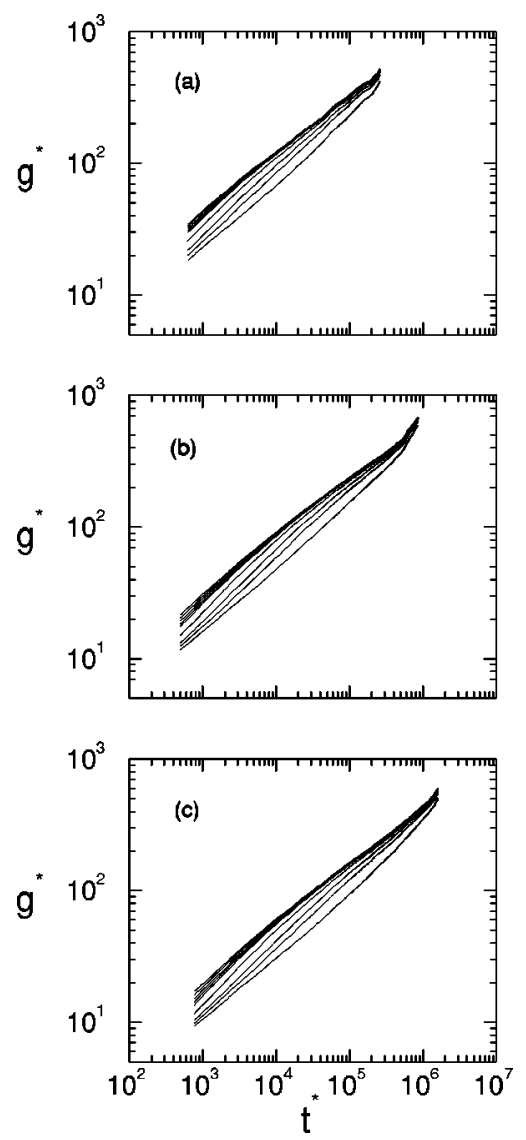

FIG. 7. Reduced mean squared displacement of end blocks vs reduced time for chains of length 192 at (a) $\phi=0.40$, (b) $\phi=0.45$, and (c) $\phi=0.50$. Included are the mean squared displacements for end blocks containing 1, 2, $3,4,5,10,20,32$, and 64 segments (on each side of the chain), from top to bottom.
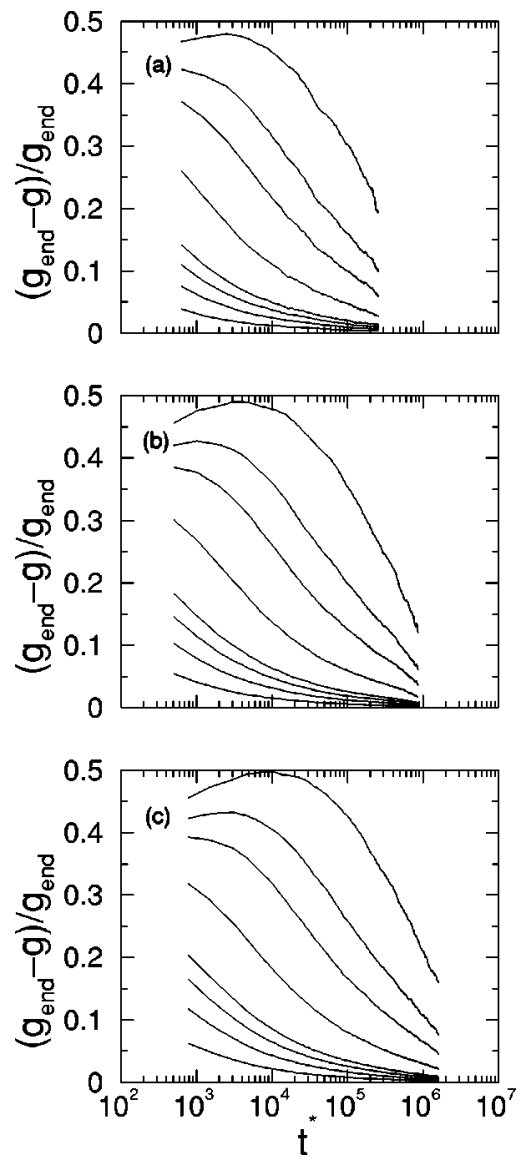

FIG. 8. Fractional difference between the mean squared displacement of different sized end blocks and that of the reference end block containing one segment (on each chain end) vs reduced time for the chains of length 192 at (a) $\phi=0.40$, (b) $\phi=0.45$, and (c) $\phi=0.50$. Included are the fractional differences for end blocks containing 2, 3, 4, 5, 10, 20, 32, and 64 segments (on each side of the chain), from bottom to top.

$=0.40$, (b) $\phi=0.45$, and (c) $\phi=0.50$. The end block sizes are $1,2,3,4,5,10,20,32$, and 64 segments (on each side of the chain), from top to bottom. Most of the mean squared displacement curves are different than that of the smallest end block containing one segment. The ability of the different sized end blocks to mimic the behavior of the end segments depends on the time range of interest. At the early times, the largest difference between the mean squared displacements of the different sized end blocks is observed. As time increases, the mean squared displacements of the different sized blocks approach the same curve.

From the mean squared displacement curves for the different sized end blocks, it appears that very small end blocks are needed to minimize the deviation between the end block behavior and that of the endmost segments. To determine the extent of the deviation between the different sized end blocks, the fractional difference between the mean squared displacement of each sized block and that of the smallest block containing one segment was calculated and analyzed.

Figure 8 displays the fractional difference between the mean squared displacement of different sized end blocks and that of the reference end block containing one segment as a function of reduced time for chains of length 192 at (a) $\phi$ $=0.40$, (b) $\phi=0.45$, and (c) $\phi=0.50$. The curves, from bot- 
TABLE IV. Maximum fractional difference for the different sized end blocks. ${ }^{a}$

\begin{tabular}{ccccccccc}
\hline \hline & \multicolumn{7}{c}{ Number of segments (on each side) } \\
\cline { 2 - 9 }$\phi$ & 2 & 3 & 4 & 5 & 10 & 20 & 32 & 64 \\
\hline 0.40 & 0.0389 & 0.0755 & 0.1098 & 0.1416 & 0.2615 & 0.3721 & 0.4232 & 0.4799 \\
0.45 & 0.0547 & 0.1035 & 0.1464 & 0.1836 & 0.3014 & 0.3854 & 0.4278 & 0.4905 \\
0.50 & 0.0629 & 0.1180 & 0.1649 & 0.2042 & 0.3181 & 0.3927 & 0.4319 & 0.4965 \\
\hline \hline
\end{tabular}

${ }^{a}$ The maximum fractional differences between the mean squared displacements of different sized end blocks and that of the segment on each chain end are reported. The different sized end blocks include 2, 3, 4, 5, 10, 20, 32, and 64 segments on each chain end.

tom to top, are for the fractional differences for the end blocks containing $2,3,4,5,10,20,32$, and 64 segments on each chain end. None of the blocks exhibit a fractional difference of zero when compared to the end segments. At early times, $t \leqslant \tau_{e}$, the fractional differences for the different sized blocks are large. All of the different sized end blocks, except the two largest (those containing 32 and 64 segments), exhibit their maximum fractional difference at the initial time shown. The two largest end blocks reach their maximum fractional difference at times around $\tau_{e}$. As time increases further, the fractional differences of all of the different sized blocks decrease. The maximum value in the fractional difference provides a measure of the different sized end blocks' ability to mimic the behavior of the end segments.

Table IV lists the maximum fractional difference between the mean squared displacement of the different sized end blocks and that of the reference end block containing one segment. The values are largest for the chains at the largest volume fraction of $\phi=0.50$. This indicates that more of the chain is confined, and thus fewer segments exhibit end behavior at larger volume fraction. For any given volume fraction, the maximum fractional difference increases with increasing end block size as expected. The maximum fractional difference is only less than 0.1 for the end blocks containing two segments at all volume fractions and for the end block containing three segments at the lowest volume fraction of $\phi=0.40$.

\section{Dynamics of segment relaxation along the chain}

In this section, we study the dynamics of segment relaxation along the chain. The aim is to determine how segment relaxation propagates along the length of the chain with increasing time. This information is helpful for determining the mechanism of chain relaxation and, thus, provides information about entanglement relaxation.

\section{Mean squared displacements of small blocks of segments at various positions along the chain}

The mean squared displacements of small blocks of segments at various positions along the chain were analyzed to determine how segment position affects the time when entanglement relaxation occurs and hence obtain information about the mechanism of entanglement relaxation and release. The tube model predicts that tube relaxation starts at the chain ends and progresses along the chain toward the chain middle where final relaxation occurs. As this relaxation occurs, the ends of the chain escape the tube and become part of the two minor chains (one on each chain end). The minor chains are the end portions of the chain which have relaxed. The minor chain length increases with time as the relaxation progresses along the chain length. When the mean squared displacement of a particular block increases more than that of the middle block, the segments in that block have relaxed and become part of a minor chain. We use the time when this relaxation occurs to determine how the minor chain length increases with increasing time.

Figure 9 displays the mean squared displacements of blocks of segments at various positions along the chain as a function of reduced time for chains of length 192 at (a) $\phi$ $=0.40$, (b) $\phi=0.45$, and (c) $\phi=0.50$. The mean squared displacement curves for nine different blocks of segments at
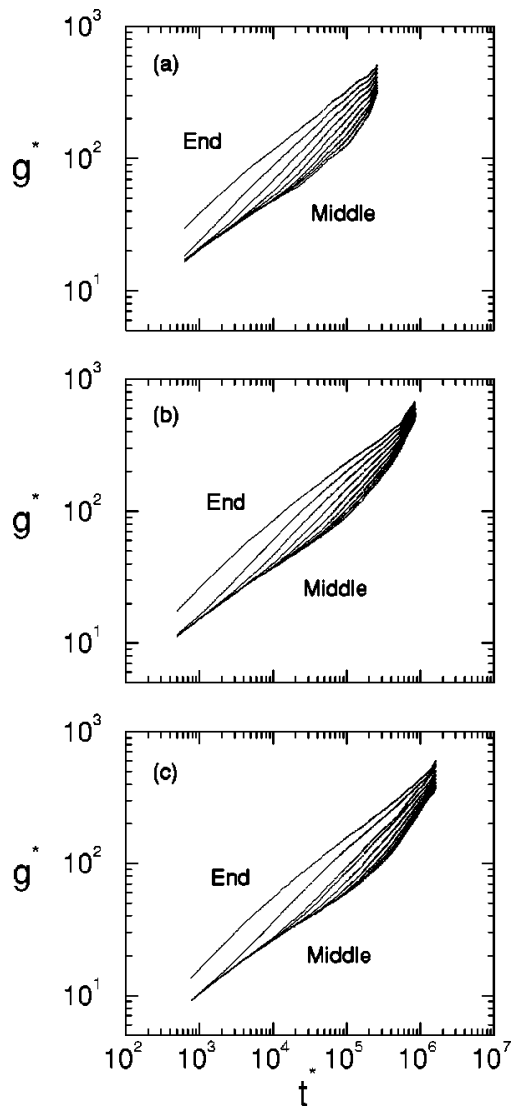

FIG. 9. Reduced mean squared displacement vs reduced time for chains of length 192 at (a) $\phi=0.40$, (b) $\phi=0.45$, and (c) $\phi=0.50$. The top curve is for the five outer segments at each chain end. The next seven curves are for blocks of segments at intermediate positions along the chain. The bottom curve is for the ten inner segments in the chain middle. 
various positions along the chain are included. The top curve is for the 5 outer segments (segments $1-5$ and 188-192). The next seven curves are for blocks of segments at intermediate positions along the chain and include the following groups of 10 segments: segments $11-15$ and 178-182, $21-25$ and $168-172,31-35$ and $158-162,41-45$ and $148-$ $152,51-55$ and $138-142,61-65$ and $128-132$, and $71-75$ and $118-122$. The bottom curve is for the 10 inner segments (segments 92-101).

At the earliest times shown in Fig. 9, all of the blocks, except the one containing the endmost segments (top curve), have moved approximately the same distance. This indicates that chain connectivity is the dominant factor during this time range, as the tube model dictates. The end block is able to move more than the other blocks because the end segments are bound on only one side while the other segments are bound on both sides. As time increases, the various mean squared displacements begin to fan out as intermediate blocks containing segments near the chain ends experience more relaxation than the inner segments, as indicated by the increase in slope of the mean squared displacement curve. First, the block that includes segments 11-15 and 178-182 (second curve from top) begins to relax. This relaxation then progresses to the next block (segments 21-25 and 168-172) along the chain closer to the chain middle and continues with the next sections in order toward the chain middle. This indicates that the initial relaxation of entanglements occurs near the chain ends and progresses toward the chain middle with time, as the tube model predicts. The time when a block relaxes is used to determine the minor chain length as a function of time as is explained below.

\section{Relaxation time of blocks at various positions along the chain}

When the mean squared displacement of a particular block increases beyond that of the middle block, the segments in that block have relaxed and become part of a minor chain. At the time when a block has relaxed, the minor chain length has increased to include the segments in that block. Therefore, the times when blocks at various positions relax are used to determine how the minor chain length increases with time. The time when the relaxation of a block occurs was determined by comparing the mean squared displacement of that block to that of the middle block.

Three different methods were used to obtain an average value for each block's relaxation time. These values were then used in the minor chain investigation. First, the relaxation time of a block was estimated as the time when its mean squared displacement curve experiences a large increase in slope compared to that of the middle block, causing its mean squared displacement to increase above that of the middle block. Next, the actual data points were included with the mean squared displacement curves, and the relaxation time of a block was estimated as the time of the data point closest to the time when the block's mean squared displacement curve experienced a large increase in comparison to that of the middle block. Finally, the fractional difference between the mean squared displacement of each block and that of the middle block was calculated and plotted versus
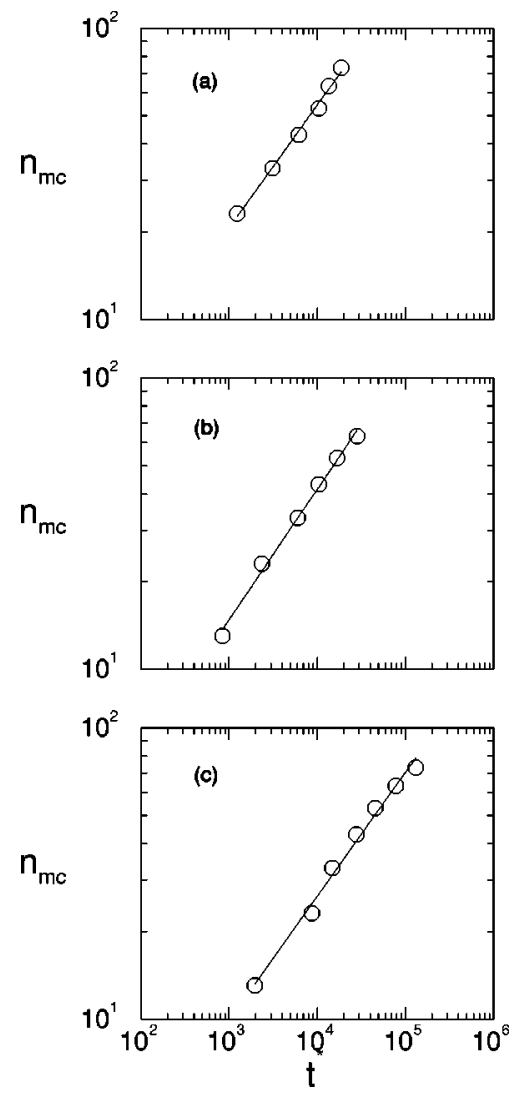

FIG. 10. Minor chain length vs reduced time for chains of length 192 at (a) $\phi=0.40$, (b) $\phi=0.45$, and (c) $\phi=0.50$. The circles represent the estimated relaxation times and minor chain lengths. The lines correspond to the power law fit of the estimated data points.

time on a logarithmic scale. The relaxation time was estimated as the time of the data point at which the fractional difference began to increase. The three relaxation times for each block along the chain were averaged to obtain the (arithmetic mean) relaxation time for each block. For each block along the chain, the number of segments in the minor chain was estimated based on the average position of the segments in the block. These estimated relaxation times and minor chain lengths were used to compare the simulation results to the minor chain reptation model predictions, as discussed below.

\section{Minor chain length as a function of time}

The behavior of the estimated minor chain lengths as a function of time was determined in order to obtain more information about entanglement relaxation and to compare the simulation results to theoretical predictions. The minor chain reptation model predicts that the number of segments in the minor chain, $n_{\mathrm{mc}}$, exhibits power law scaling with time so that $n_{\mathrm{mc}} \sim t^{0.5} \cdot{ }^{31,32}$ We obtain a power law fit to the estimated minor chain lengths versus time to determine how well our simulation results fit this trend.

Figure 10 displays the estimated minor chain length versus time for chains of length 192 at (a) $\phi=0.40$, (b) $\phi$ $=0.45$, and (c) $\phi=0.50$. The circles represent the estimated data points that were obtained from the mean squared displacements. The estimated minor chain lengths are the aver- 
age segment positions of the small blocks, and the estimated times are the average times when those blocks became more relaxed than the middle blocks as determined from the mean squared displacements. The lines correspond to a power law fit to the estimated data points.

The estimated minor chain lengths versus time of all of the systems essentially fall on a straight line in the $\log -\log$ plot, indicating that the minor chain length follows a power law with time. The power law fits $\left(n_{\mathrm{mc}} \sim t^{x}\right)$ to the data for the different systems provide similar exponents: $x \approx 0.42$ $\pm 0.03,0.45 \pm 0.03$, and $0.42 \pm 0.03$, at $\phi=0.40,0.45$, and 0.50 . These exponents are somewhat less than the predicted value of 0.5 . The relaxation of chain segments progresses from the chain ends toward the chain middle, and the minor chain lengths exhibit power law behavior with time. However, the lower than predicted exponents suggest that the relaxation of the segments along the chain progresses somewhat more slowly than the minor chain reptation model predicts.

\section{CONCLUSIONS}

In this study, discontinuous molecular dynamics simulations were performed to investigate how a segment's position along the chain affects its mean squared displacement. First, we studied how the size of the blocks of segments considered affects the difference between the dynamics of end and middle segments. Then, we determined what portion of the chain exhibits chain middle behavior and what portion exhibits chain end behavior. Finally, we investigated the dynamics of segment relaxation along the chain to ascertain how the relaxation propagates along the chain.

We studied how the size of the block considered affects conclusions about the difference between the dynamics of end and middle segments of chain molecules. The aim was to determine if large blocks, like those used during experiments, are able to distinguish the dynamics of chain ends from that of the chain middle. First, we analyzed the mean squared displacements of chain end segments, chain middle segments, and the whole chain for triblock chains. For the triblock case, we found that the mean squared displacements of the end blocks, the middle blocks, and the whole chain exhibit similar values, and that the greatest difference occurred during the intermediate times sampled. Next we analyzed the mean squared displacements of chain end segments, chain middle segments, and the whole chain for the case where just ten segments were used to represent the chain end or the chain middle. For the small-block case, we found that there was a larger difference between the three mean squared displacement curves than there was for the triblock case. Finally, we compared the results from the triblock and small-block cases directly. There was a large difference between the end block mean squared displacements from the triblock and small-block cases. However, the middle-block mean squared displacements were essentially the same in the two cases. The ratio of the end block mean squared displacement to the middle-block mean squared displacement was calculated for the different cases to determine the difference between the dynamics of the end and middle blocks. It was found that the small blocks were needed to capture the difference between the end and middle-block mean squared displacements.

The study on the dynamics of different sized blocks was extended to include other size blocks to determine what portion of the chain exhibited chain middle behavior and what portion exhibited chain end behavior. The aim was to determine what size blocks are sufficient for studying the middle section or end section mean squared displacements. From the mean squared displacements of different sized middle blocks, it was found that very large middle blocks provide similar results to very small middle blocks. This indicates that a large portion of the chain exhibits chain middle behavior. On the other hand, the mean squared displacements of different sized end blocks indicated that small end blocks are needed to obtain end segment behavior. Therefore, a small portion of the chain exhibits chain end behavior.

The dynamics of segment relaxation along the chain were studied to determine how segment relaxation propagates along the length of the chain with increasing time. First, the mean squared displacement versus time was analyzed for small blocks of segments at various positions along the chain. The relaxation of these blocks started at the chain ends and progressed toward the chain middle with time, as the tube model predicts. The time when the relaxation of a block occurred was estimated by determining when the block's mean squared displacement increased above that of the middle block. These estimated relaxation times represented the time at which the minor chain included segments along the chain up to the middle of that block. The minor chain length versus time exhibited the power law behavior $n_{\mathrm{mc}} \sim t^{x}$, where $x \approx 0.42 \pm 0.03,0.45 \pm 0.03$, and $0.42 \pm 0.03$ at $\phi=0.40,0.45$, and 0.50 . This time dependence was slightly lower than the value $x=0.5$ predicted by the minor chain reptation model.

\section{ACKNOWLEDGMENTS}

We would like to thank J. van Zanten for helpful discussions. This work was supported by a National Science Foundation Graduate Research Fellowship, a DuPont Graduate Fellowship, an Eastman Graduate Fellowship, and the Office of Energy Research, Basic Sciences, Chemical Science Division of the U.S. Department of Energy under Contract No. DE-FG02-97-ER14771. Acknowledgment is made to the Donors of the Petroleum Research Fund administered by the American Chemical Society for partial support of this work. We thank the North Carolina Supercomputing Center for grants of computing time and file storage space.

${ }^{1}$ P. E. Rouse, J. Chem. Phys. 21, 1272 (1953).

${ }^{2}$ P. G. de Gennes, J. Chem. Phys. 55, 572 (1971).

${ }^{3}$ M. Doi and S. F. Edwards, The Theory of Polymer Dynamics (Oxford University Press, Oxford, 1986).

${ }^{4}$ B. Ewen, D. Richter, and B. Farago, Acta Polym. 45, 143 (1994).

${ }^{5}$ K. Welp, R. Wool, S. Satija, S. Pispas, and J. Mays, Macromolecules 31, 4915 (1998).

${ }^{6}$ K. Welp, R. Wool, G. Agrawal, S. Satija, S. Pispas, and J. Mays, Macromolecules 32, 5127 (1999).

${ }^{7}$ W. Paul, K. Binder, D. W. Heermann, and K. Kremer, J. Chem. Phys. 95, 7726 (1991). 
${ }^{8}$ T. Kreer, J. Baschnagel, M. Muller, and K. Binder, Macromolecules 34, 1105 (2001).

${ }^{9}$ J. Skolnick and A. Kolinski, Adv. Chem. Phys. 78, 223 (1990).

${ }^{10}$ K. Kremer and G. S. Grest, J. Chem. Phys. 92, 5057 (1990).

${ }^{11}$ B. Dunweg, G. S. Grest, and K. Kremer, in Numerical Methods for Polymeric Systems, edited by S. G. Whittington (Springer-Verlag, New York, 1998).

${ }^{12}$ M. Pütz, K. Kremer, and G. S. Grest, Europhys. Lett. 49, 735 (2000).

${ }^{13}$ S. W. Smith, C. K. Hall, and B. D. Freeman, Phys. Rev. Lett. 75, 1316 (1995).

${ }^{14}$ S. W. Smith, C. K. Hall, and B. D. Freeman, J. Chem. Phys. 104, 5616 (1996).

${ }^{15}$ S. W. Smith, C. K. Hall, B. D. Freeman, and J. A. McCormick, in $\mathrm{Nu}$ merical Methods for Polymeric Systems, edited by S. G. Whittington (Springer-Verlag, New York, 1998).

${ }^{16}$ A. Kolinski, J. Skolnick, and R. Yaris, J. Chem. Phys. 86, 1567 (1987).

${ }^{17}$ J. A. McCormick, C. K. Hall, and S. A. Khan, Macromolecules (to be published).

${ }^{18}$ K. S. Schweizer, J. Chem. Phys. 91, 5822 (1989).

${ }^{19}$ M. Doi and S. F. Edwards, J. Chem. Soc., Faraday Trans. 2 74, 1789 (1978).
${ }^{20}$ S. W. Smith, C. K. Hall, and B. D. Freeman, J. Comput. Phys. 134, 16 (1997).

${ }^{21}$ P. J. Flory, Statistical Mechanics of Chain Molecules (Interscience, New York, 1969).

${ }^{22}$ D. C. Rapaport, J. Phys. A 11, L213 (1978).

${ }^{23}$ A. Bellemans, J. Orban, and D. V. Belle, Mol. Phys. 39, 781 (1980).

${ }^{24}$ B. J. Alder and T. E. Wainwright, in Symposium on Transport Processes in Statistical Mechanics, edited by I. Prigogine (Interscience, New York, 1956).

${ }^{25}$ B. J. Alder and T. E. Wainwright, J. Chem. Phys. 31, 459 (1959).

${ }^{26}$ J. M. Haile, Molecular Dynamics Simulation: Elementary Methods (Wiley, New York, 1992).

${ }^{27}$ M. P. Allen and D. J. Tildesley, Computer Simulation of Liquids (Clarendon, Oxford, 1987).

${ }^{28}$ D. C. Rapaport, J. Comput. Phys. 34, 184 (1980).

${ }^{29}$ J. J. Erpenbeck and W. W. Wood, in Modern Theoretical Chemistry, edited by B. J. Berne (Plenum, New York, 1977).

${ }^{30}$ Y. Zhou, S. W. Smith, and C. K. Hall, Mol. Phys. 86, 1157 (1995).

${ }^{31}$ Y. H. Kim and R. P. Wool, Macromolecules 16, 115 (1983).

${ }^{32}$ R. P. Wool, Polymer Interfaces; Structure and Strength (Hanser, Munich, Germany, 1995). 